\title{
Adaptive Empirical Mode Decomposition for Bearing Fault Detection
}

\author{
Van Tuan Do* - Le Cuong Nguyen \\ Electric Power University, Department of Electronics and Telecommunication, Vietnam
}

\begin{abstract}
Many techniques for bearing fault detection have been proposed. Two of the most effective approaches are using envelope analysis and the empirical mode decomposition method (EMD), also known as Hilbert-Huang transform (HHT), for vibration signals. Both approaches can detect the bearing fault when the vibration data are not strongly disturbed by noise. In the approach using EMD method, the EMD algorithm is used to decompose the vibration data into components with a well-defined instantaneous frequency called intrinsic mode functions (IMFs). Then a spectral analysis is used for selected IMFs to indicate the appearance of nominal bearing defect frequencies (nominal frequencies), which are caused by bearing faults. However, when the data are strongly disturbed by noise and other sources, the approach can be failed. The EMD algorithm generates IMFs itself; hence, the IMFs will also contain both a fault signal part and other components. It becomes more severe when the other components are dominant and have significant amplitudes near the same frequencies as the fault signal part. Moreover, in the IMF extracting process, the EMD methods keeps removing the low-frequency components until the residual is an IMF; therefore, until the IMF is found, some of the fault signal parts can be removed and will appear in the next IMFs. Therefore, it must be emphasized that the energy of the fault signal part can spread in some IMFs that will lead the detecting faulty features in any of those IMFs to be weak. In this paper, we address the weakness of the EMD method for bearing fault detection by introducing an adaptive EMD (AEMD). The AEMD algorithm is intended to generate IMFs so that one of them contains most of the energy of the fault signal part; thus, it assists our model to detect the bearing fault better. Moreover, the bearing fault detection model using the AEMD method with simulation data is compared with those of using envelope analysis and the latest version of the EMD, called an ensemble EMD algorithm. An application study of bearing fault detection with AEMD method is also carried out.
\end{abstract}

Keywords: bearing fault detection, Hilbert-Huang transforms, empirical mode decomposition, intrinsic mode function, envelope analysis, nominal frequency

Highlights

- $\quad$ Bearing faults in the low-speed bearing system are hard to detect with the original EMD algorithm as well as the envelope analysis.

- $\quad$ By considering the energy of the IMF, the proposed adaptive EMD algorithm works well in bearing fault detection and performs better than the original EMD algorithm.

\section{O INTRODUCTION}

There have been a huge number of studies on rotating machine fault detection, especially for bearings, which are main components of the inner ring, outer ring, bearings (rolling elements) and cage. There are a number of mechanisms leading to bearing failures such as fatigue damage, wear damage, plastic deformation, corrosion, mechanical damage, and lubrication deficiency [1] to [3]. The most common faults on bearing components are fatigue damage including wear, pitting, and spalling. Such damage is a fatigue phenomenon that occurs when a fatigue crack initiates at the surface of bearing's components or at a small depth below the surface. The crack usually propagates for a short distance in a direction roughly parallel to the surface before turning or branching to the surface. As cracks progress further into the surface, the rate of propagation increases. When the cracks have grown to the extent that they separate a piece of the surface material, a pit is formed. The material is torn away from the damaged area by the relative motion of the contacting part. A loss of material that reaches some level is frequently termed spalling [4].

When fatigue damage occurs on a bearing's component and this defective component is in contact with another component at the damage point, an impulsive force is incurred that causes the bearing to vibrate. The vibration signal obtained from the bearing consists of an impulse whenever a rolling element passes over the damage point, which can be in the outer race, inner race, or the surfaces of rolling elements or cage. The pulse appearance frequencies are dependent on the damage point locations and are called nominal bearing defect frequencies (or, more briefly nominal frequency) (or characteristic defect frequency (CDF) as called in [5]). The nominal frequencies corresponding to bearing components are calculated by Eqs. (1) to (4). The vibration signal from each pulse presents the ringing of the bearing at its resonance frequency, also called ringing pulse frequency. The pulse occurs with high amplitude 
and then the amplitude attenuates rapidly. This will cause the appearance of ringing pulse frequency and its sidebands in the vibration data. The sidebands are results of the modulation between the ringing pulse frequency and nominal frequency.

Ball pass frequency outer race (BPFO):

$$
f_{\text {outer }}=\frac{N_{b}}{2}\left(1-\frac{B_{d}}{P_{d}} \cos \theta\right) \frac{R P M}{60},
$$

ball pass frequency inner race (BPFI):

$$
f_{\text {inner }}=\frac{N_{b}}{2}\left(1+\frac{B_{d}}{P_{d}} \cos \theta\right) \frac{R P M}{60},
$$

rolling elements' frequency (BSF):

$$
f_{\text {roller }}=\frac{P_{d}}{2 B_{d}}\left(1-\left(\frac{B_{d}}{P_{d}} \cos \theta\right)^{2}\right) \frac{R P M}{60},
$$

cage or train frequency (FTF):

$$
f_{\text {train }}=\frac{1}{2}\left(1-\frac{B_{d}}{P_{d}} \cos \theta\right) \frac{R P M}{60},
$$

where $R P M$ is the shaft rotation speed, $N_{b}$ is the number of the balls or rollers, $B_{d}$ is the ball or roller diameter, $P_{d}$ is the bearing pitch diameter and $\theta$ is the contact angle.

The bearing fault detection problems are generally concerned with algorithms and models in order to detect increased vibration amplitudes at the nominal frequencies. The envelope analysis is a well-known and easy method for bearing fault detection and reported in various research, such as in [1]. This paragraph concentrates on the stateof-the-art techniques that use the EMD method for fault detection of rotating machines, especially for bearings. In [5] and [6], the authors suggested using an empirical mode decomposition (EMD) method [7] for bearing fault detection. The EMD algorithm was used to decompose the vibration signal into intrinsic mode functions (IMFs). The authors used a fast Fourier transform (FFT) amplitude spectrum for each IMF and found that the defect frequency (nominal frequency) appeared in the FFT spectrum of the first IMF. In general, when the vibration data are weakly disturbed, the method will work well. However, if the data are influenced by noise or other frequency components, the method can fail to perform adequately. For example, the fault signal part can be influenced by other rotating frequencies, sensor noise, and sensor resonant frequencies, which will be demonstrated in the later sections of this paper.
Since [5] and [6] were reported, several proposed methods utilizing the EMD algorithm have been for bearings and other rotating machines. In [8], the authors proposed a diagnosis method based on artificial neural networks (ANN). The entropy of each IMF was estimated to determine which IMF signal should be selected for the training process of the ANN network. The classification method using ANN was established to classify different bearing conditions: normal, outer race and inner race faults. In [9], the authors showed that instead of using entropy energy, which was not useful for non-stationary vibration signals, they introduced a new feature called IMF energy moment. They used the wavelet package decomposition (WPD) to de-noise the vibration data and then calculated each IMF energy component. The IMF containing the highest IMF energy component will be selected to extract faulty features mostly related to defect frequencies such as $1 \mathrm{X}, 2 \mathrm{X}$, and its harmonics. The features were then served as inputs to a back propagation network. The network was employed as a classification model for machine fault diagnosis. In [10], the authors utilized the EMD algorithm to decompose vibration data from defect roller bearings into IMFs. The autogressive models (AR) were established for each IMF to extract AR parameters and residual variations, which were then aggregated into one feature vector. A proposed diagnosis model with the Mahalanobis distance of feature vectors was used to recognize bearing faults in three conditions: normal, outer race and inner race damage. The authors in [11] explained that using the IMFs alone for rotating machine fault detection could not work well with noisy vibration data. They combined some of the consecutive IMFs into one and called it a combined mode functions (CMD) and utilized it for the fault detection of generators. If there was a sudden change in the waveform of the next IMF, then the CMDs was suggested be used more than the original IMFs. Recently, an approach called ensemble EMD (EEMD) [12] was proposed to solve the mode mixing problem that happens in the original EMD method by adding white noise to the IMFs during the decomposition process of the original EMD. The authors in [13] showed that it was not necessary to add white noise to all the decomposition modes since the low-frequency part will be affected by noise, worsening the EMD process. It also brings a higher computational complexity to the algorithm. Therefore, they simply suggested using band-limited noise instead of white noise into the signal to be decomposed. Other than proposed research with EMD for bearing fault detection, there are also several 
approaches, such as in [14] to [16]. In [14] the authors converted one dimension of the vibration signal into a two-dimension domain, and then a recognition model is applied for fault classification including bearing faults. In [15], a Meyer-Wavelet-Packets algorithm was used to detect the bearing faults and the author stated that the proposed approach provided better results than that of using coif4 wavelet. In [16], the discrete wavelet transforms and an adaptive resonance theory neural network were used to detect the gear crack. This method can be applied for bearing faults as well.

Some adaptive techniques using Morelet wavelets were successfully proposed for rotating machine component fault detection, such as [17] and [18]. In this paper; an adaptive EMD is proposed; however, two major topics are addressed: (i) improving of the original EMD algorithm in order to enhance the appearance of the nominal frequencies, and (ii) proposing a model for both simulation and experiment data for bearing fault detection. The EMD algorithm will be modified so that the output IMFs will only be adapted for the purpose of bearing fault detection. The method is called adaptive EMD (AEMD). The AEMD is used especially for addressing the weaknesses of the original EMD method proposed in [5] and [6] when data are strongly disturbed by other components and noise. The AEMD method is intended to be better than original EMD in term of showing the nominal defect frequency for bearing fault detection only. Please note that if the data is so clean in term of no influences to the ringing pulse frequency band, then the original and adaptive EMD will work equally well.

The rest of the paper is organized as follows. The EMD and ensemble EMD algorithms will be presented in Section 2. Section 3 is about the proposed adaptive EMD algorithm. The bearing analysis comparison with simulation data will be presented in Section 4, while a bearing fault detection application study with adaptive EMD is discussed in Section 5. The conclusion is follows in Section 6 .

\section{EMD AND ENSEMBLE EMD ALGORITHMS}

\subsection{Empirical Mode Decomposition}

The empirical mode decomposition method is a signal processing technique proposed for extracting all the oscillatory modes embedded in a signal without any requirement of stationarity or linearity of the data. The

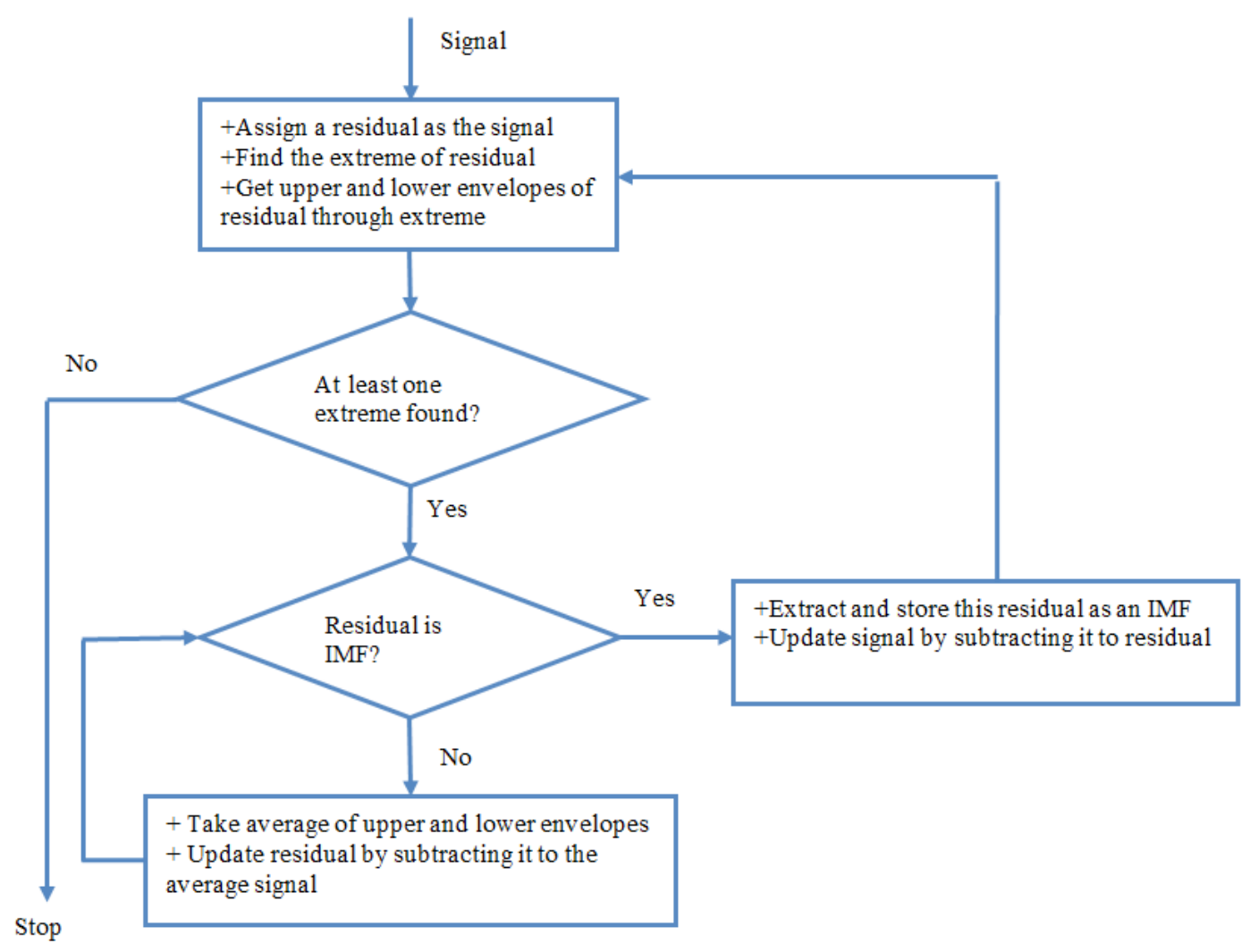

Fig. 1. Flow chart of the original EMD algorithm 
goal of this procedure is to decompose a time series into components with well-defined instantaneous frequencies by empirically identifying the physical time scales intrinsic to the data, which is the time lapse between successive extrema. Each characteristic oscillatory mode extracted, named IMF, has only one extreme (minima or maxima) between zero crossings and the mean value between the upper and lower envelope, which are established based on the extreme, is equal to zero. Therefore, in the algorithm, conditions for a signal to be an IMF are as follows:

- Differences in the number of maxima and zerocrossings must not be more than 1 .

- The mean of the average of upper and lower envelopes should be smaller than a defined threshold, which is close to zero.

The detailed algorithm can be found in [7]. The algorithm flowchart is presented in Fig. 1. The outcomes of the EMD algorithm will be several IMFs. The number of the IMFs may be different depending on the signal analysed. Among the IMFs extracted, some of them contain a fault signal part that will help identify the faults. The extracted features can be used individually or as inputs to fault diagnosis models. The bearing fault diagnosis methods using the EMD method so far deal with three problems: (i) finding the relevant IMFs; (ii) extracting relevant features, possibly related to faults from those IMFs; (iii) establishing fault detection and diagnosis models utilizing the features.

\subsection{Ensemble Empirical Mode Decomposition}

The EEMD is an improvement on the EMD algorithm targeting to remove mixing mode problems [12]. Comparing to the EMD method, the improvement of the ensemble EMD is described as follows:

- Add a white noise series to the targeted data.

- Decompose the data noise into IMFs with added white.

- $\quad$ Repeat step 1 and step 2 multiple times, but with different white noise series each time.

- Obtain the (ensemble) means of corresponding IMFs of the decompositions as the final result.

\section{ADAPTIVE EMPIRICAL MODE DECOMPOSITION}

As previously discussed, the envelope analysis is easy to implement and a method of choice for bearing analysis if a suitable band pass filter is available. However, for implementation on an automation system or embedded system, the EMD method is preferred. The indication of the nominal frequency appearance fails with the EMD method when the data are strongly disturbed, which will be indicated in the analysis result section. Looking into the decomposition process of the original EMD algorithm, the EMD method extracts the IMF with the highest frequencies first, followed by the lower frequencies. In other words, the first IMF will contain the highest frequency components, and the last one will contain the lowest frequency components. To extract an IMF, the algorithm keeps removing low-frequency components until the residual (signal after removing low-frequency components) is an IMF. The reasons the EMD method failed in the previous example can be explained as follows:

- The signal part caused by faults can easily share the energy among some IMFs if it has a highfrequency bandwidth that will lead to detecting the fault features in any of those IMFs to be weak.

- When data is complicated, it is easy for the algorithm to extract IMFs, including the other components that interfere with the fault signal part.

In the AEMD, we supplement the decomposition process of the EMD algorithm to avoid the first reason. On the process of removing low-frequency components in the EMD algorithm, the AEMD algorithm keeps calculating the energy of frequency components of the residual concentrating on the frequency segment that contains the nominal frequency and its harmonics. The energy is called "peak energy", determining whether the low frequencies are related to faults or not. The IMF will be assigned as the residual at the point that the peak energy of the residual has the highest value. If the lowfrequency components are related to faults, then they will be retained in the extracted IMF. Otherwise, they will be removed and will appear in the next IMFs. The peak energy value in this paper is calculated as the total power spectrum density at the nominal frequency and its first two harmonics. Other energy indicators can be considered to replace power spectrum density, such as the amplitude of nominal frequency and its first harmonics, the power spectrum of the nominal frequency and its first two harmonics, etc. [19]. The details of the AEMD algorithm can be seen in Fig. 2 .

The goal of a bearing fault detection model based on a vibration signal is to identify the fault symptom that is embedded into the vibration signal; here, it is the nominal frequency. Our proposed model using the proposed AEMD method is as follows:

- Use the AEMD algorithm for the vibration data to extract IMFs signals. 
- Choose the best IMF.

- Calculate the power spectrum of the envelope signal of this IMF.

- Estimate the amplitude of nominal frequency in the plots of the power spectrum.

\section{BEARING ANALYSIS DECOMPOSITION}

\subsection{Simulation Data}

In this section, we simulate the vibration data for a normal bearing and for a bearing with fatigue damage on its outer race. Whenever a rolling element goes over the damage point on the surface of the outer race, there will be a ringing pulse appearing in the bearing vibration data. The vibration data measured from the bearing will be covered by high-level imbalance and misalignment components, random vibrations accompanied by friction and other sources such as environment noise, sensor noise, and sensor resonance signals. The following frequency components are dominant in the vibration data measured by accelerometers on the surfaces of bearings: (i) shaft frequency, which is $1 \mathrm{X}$ frequency component, caused by rotor rotation; (ii) imbalance vibration (1X), its harmonics $(2 \mathrm{X}, 3 \mathrm{X}, 4 \mathrm{X})$, and its sidebands caused by misalignment components; (iii) other rotating components, such as gear-mesh frequency, sidebands, and gear rotating frequencies. Simulations of vibration data from bearings are well explained in [3] and [20].

The simulated vibration signal, $x(t)$, is generated as follows:

$$
x(t)=a(t)+n(t)+r(t),
$$

where $a(t)$ are approximation signals, $n(t)$ is Gaussian noise and $r(t)$ are ringing pulse signals. More explanation can be found in [20]. In this paper, the vibration data for outer race defect bearings are simulated as explained in Eq. (5) and without ringing pulse part, $t(t)$ for a normal bearing. The nominal

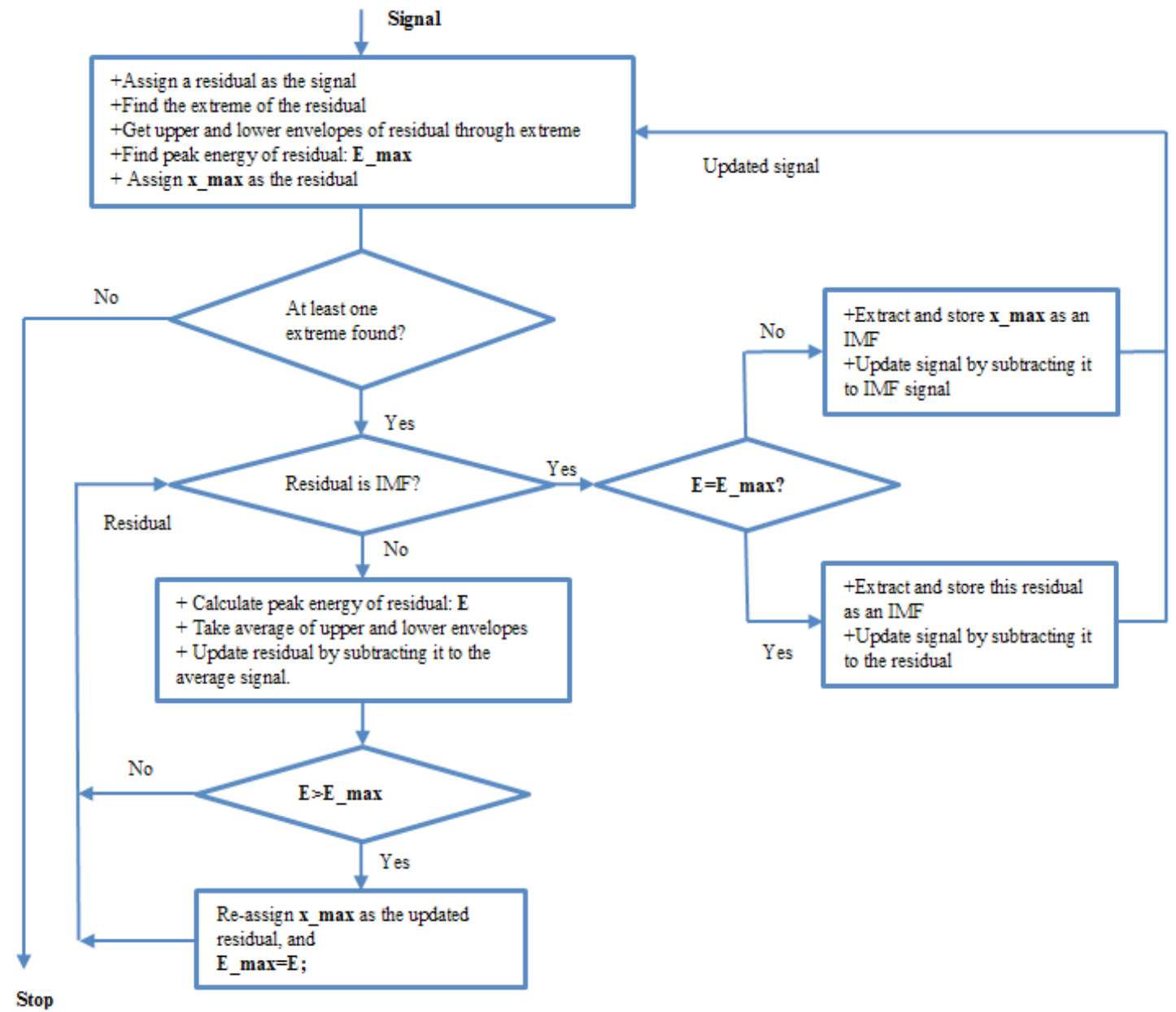

Fig. 2. Flowchart of the proposed AEMD algorithm 
frequency $f_{\text {outer }}$, is $20 \mathrm{~Hz}$ and ringing pulse frequency is $1000 \mathrm{~Hz}$.

\subsection{Bearing Fault Detection with Envelope Analysis and Original EMD Methods}

This section is aimed to show that the envelope analysis and the EMD method work when data are clean, and do not work when data are not.

The envelope analysis method [1] and [20] is used with both normal and faulty bearing simulation vibration data. The plots of the power spectrum of the signal after the envelope analysis are intuitively used to estimate the appearance of the nominal frequency of the outer race defect bearing. For the EMD method, it is used to generate the IMFs. With the nominal frequency of $20 \mathrm{~Hz}$ and ringing pulse frequency is
$1000 \mathrm{~Hz}$, the IMF \#1 (IMF1) is selected. The Hilbert analytic signal of the IMF1 is generated. Then the plots of the power spectrum of the analytic signal are intuitively used. We use both methods for normal and faulty bearing data, which are supposed to indicate the appearance of the nominal frequency $(20 \mathrm{~Hz})$ in power spectrum plots for faulty bearing signal but do not for normal bearing data.

In Fig. 3, the differences between the normal and faulty bearing can be seen in Fig. 3b in Region I where the ringing pulse frequency components (around $1000 \mathrm{~Hz}$ ) occur. Note that the ringing pulse frequency components only appear when the bearing fault is present. With this example, the $f_{\text {outer }}(20 \mathrm{~Hz})$ and its harmonics are clearly shown in the Figs. 3c and $\mathrm{d}$. This proves that both envelope analysis and EMD methods work well for clean vibration data.
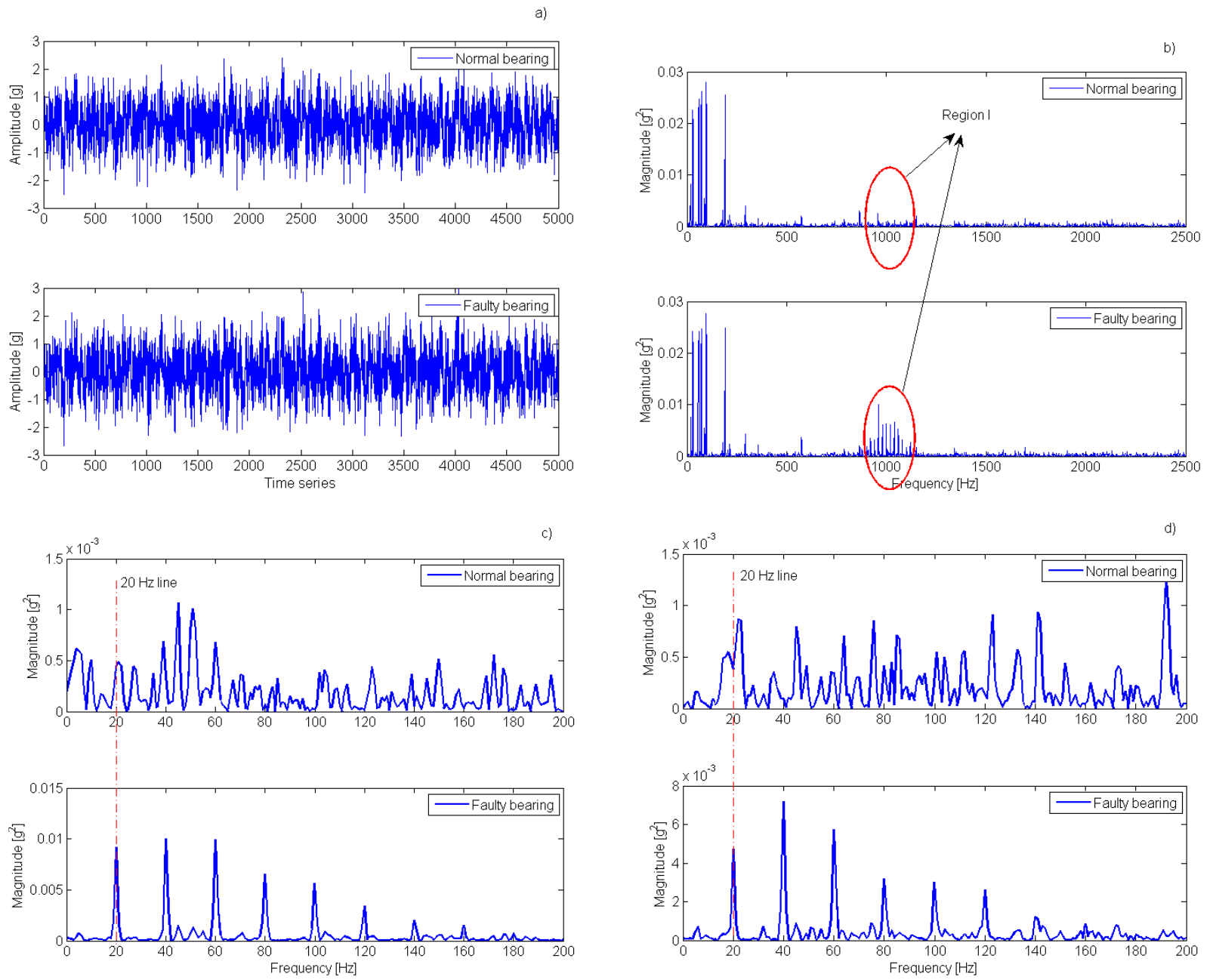

Fig. 3. Simulation of bearing detection with envelope analysis and EMD method with clean vibration data; a) waveform of perfect and faulty bearing signal, b) power spectrum of perfect and faulty bearing signal, c) power spectrum of the outcomes with envelope analysis; here, the band-pass filtering for [ $500 \mathrm{~Hz}$ to $1500 \mathrm{~Hz}$ ] is applied in envelope analysis, and d) power spectrum of envelope signal of first IMF 

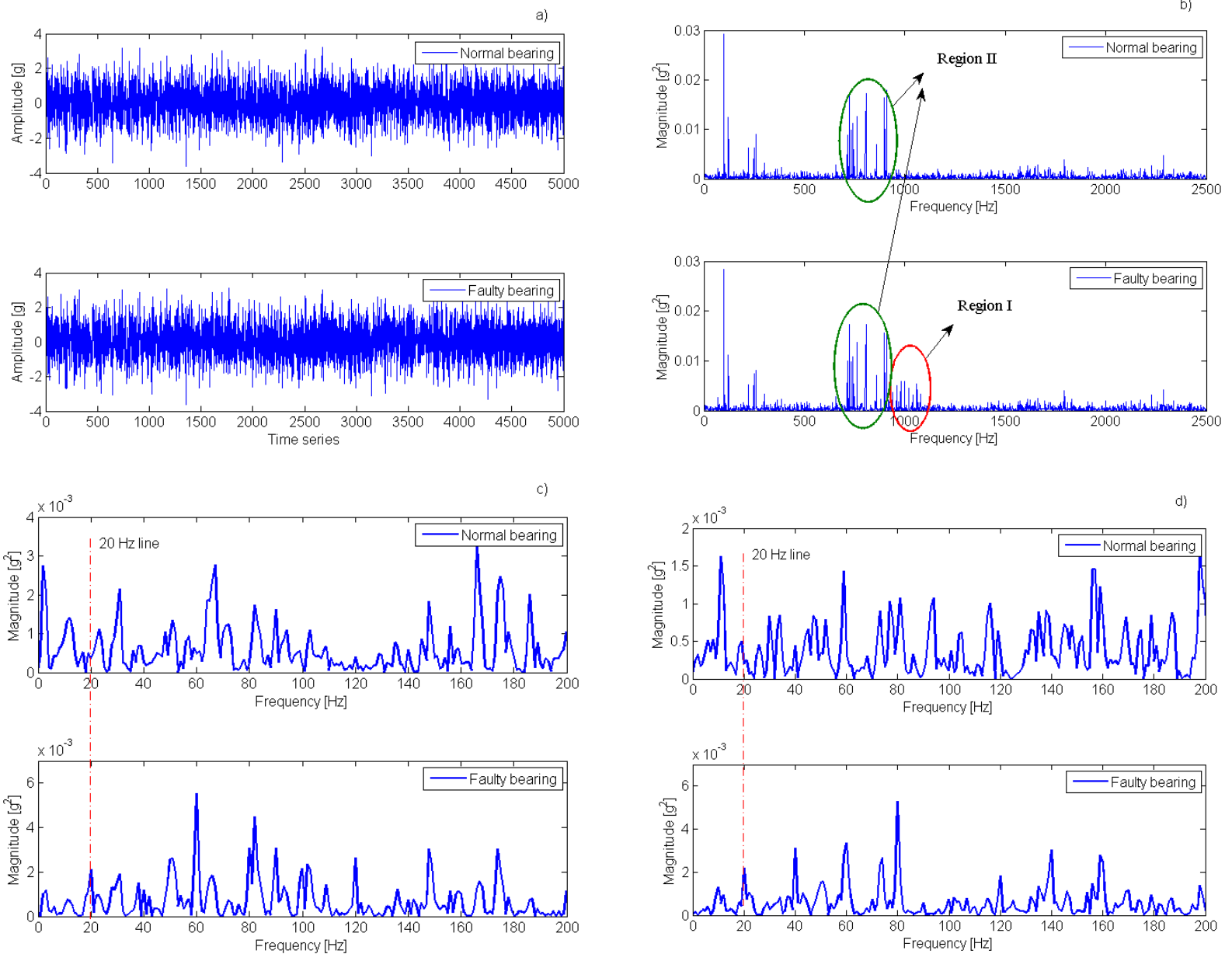

Fig. 4. Simulation of bearing detection with envelope analysis and EMD method with disturbed vibration data, the disturbance is Region II; a) waveform of perfect and faulty bearing signal, b) power spectrum of perfect and faulty bearing signal, c) power spectrum of the outcomes with envelope analysis; here, the band-pass filtering for [500 Hz-1500 Hz] is applied in envelope analysis, and d) power spectrum of envelope signal of first IMF

In Fig. 4, the differences between normal and faulty bearings are only in Region I; however, Region II close to region I which contains dominant components. As discussed before, Region II would cause both the envelope analysis and EMD to not work well. Indeed, looking at Fig. $4 \mathrm{c}$ and d, we are unable to emphasize the nominal defect bearing frequency, $f_{\text {outer }}(20 \mathrm{~Hz})$. Region II interfered with the outcomes of the both methods.

\subsection{Comparison of Envelope Analysis, EEMD, and AEMD with Simulation Data}

In this section, the bearing fault detection model provided in section 3 using AEMD will be compared with those of using EEMD and envelope analysis. The simulated data for an outer race defect bearing above

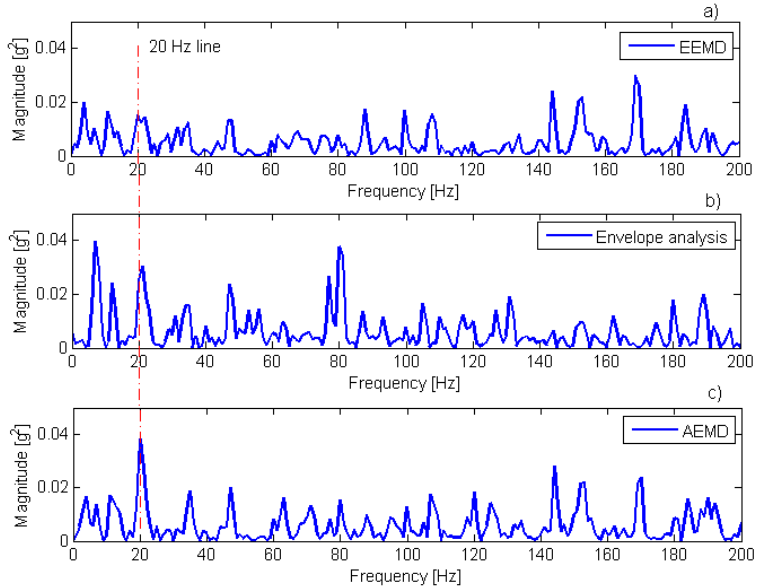

Fig. 5. Comparison the power spectrum with three methods: a) EEMD, b) envelope analysis and c) adaptive EMD with simulation data 
is used. Fig. 5 shows the average power spectrum of 10 trials. The plots show that the amplitude of the nominal frequency with adaptive EMD is higher than that of with the EEMD and envelope analysis. It is also the highest peak found in the plots. The amplitude even cannot be recognized with EEMD method since it is not dominant and weak in comparison to other peaks in the plot. With envelope analysis, the amplitude is relatively high but not the most dominant one in the plot.

The performance of the AEMD method is intuitively better than ensemble EMD method and envelope analysis in terms of indicating the nominal defect frequency $(20 \mathrm{~Hz})$.

\section{APPLICATION STUDY FOR BEARING FAULT DETECTION WITH THE AEMD METHOD}

The test rig consisted of a genuine railway wagon bearing structure, where both the supporting bearings and test bearings were two-row roller bearings (SKF 229750). The shaft rotation speed could be adjusted between $0 \mathrm{rpm}$ to $100 \mathrm{rpm}$. Furthermore, the loading of the test bearing was adjustable. For the test measurements, the rig was supplied with a bearing with a minor outer race defect as shown in Figs. 6a and b. In Fig. 6c, on the left are the two adjacent supporting bearings, the damaged bearing to be tested is in the middle, and on the right there are another two adjacent supporting bearings. The bearing parameters are provided in Table 1.

The experiment was carried out at a sampling rate of $4000 \mathrm{~Hz}$, rotating of the input shaft of $79 \mathrm{rpm}$, and a load of $78 \mathrm{kN}$. Vibration data collected from vertical and horizontal accelerometers have a duration of 60 seconds and from both normal and outer race defect bearings.

Table 1. Bearing parameters

\begin{tabular}{lc}
\hline Number of rolling elements & 22 \\
\hline Rolling element diameter & $20 \mathrm{~mm}$ \\
\hline Bearing pitch diameter & $180 \mathrm{~mm}$ \\
\hline Contact angle & 18 degrees \\
\hline
\end{tabular}

With bearing parameters provided in Table 1, the nominal frequencies $f_{\text {outer }}$ are $13 \mathrm{~Hz}$ calculated by Eq. (1).

In this experiment, the bearing parameters are known. To identify the fault happening on the outer race of the bearing, the bearing fault detection model must be able to indicate the appearance of the nominal frequency in the vibration data. This section is intended to use the proposed model mentioned

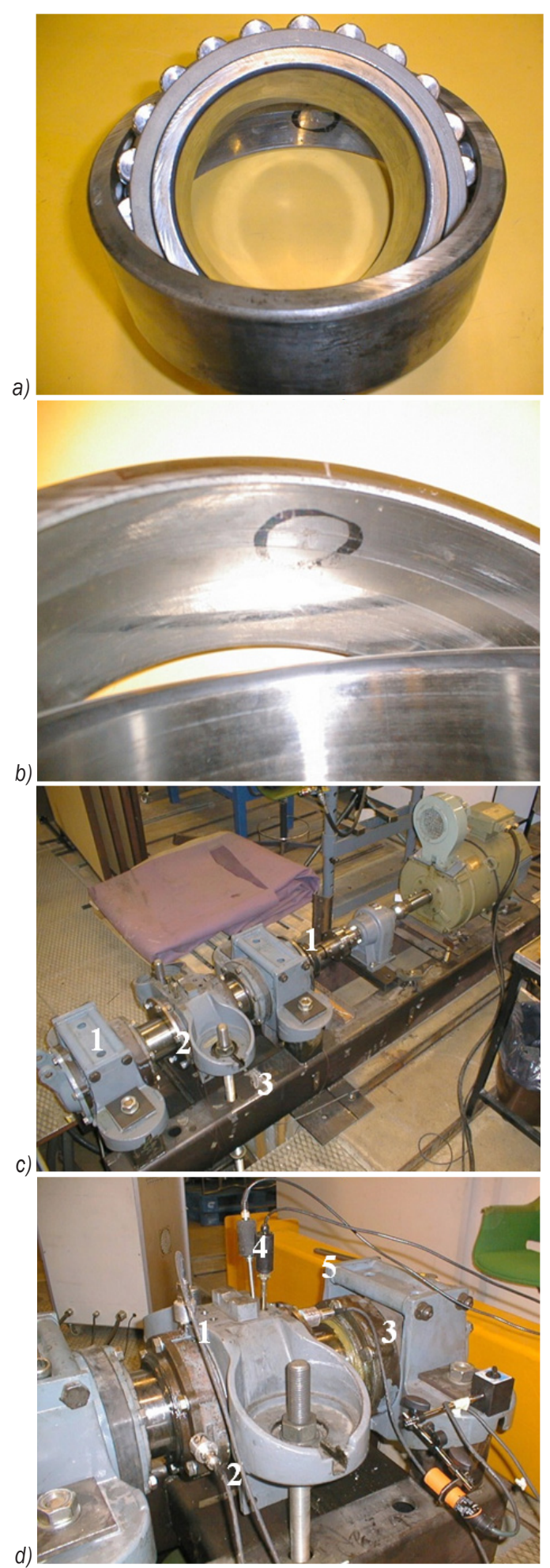

Fig. 6. a) Location of the outer race damage; b) closer look at the damage; c) test rig; 1 supporting bearings, 2 test bearing, 3 load adjusting nut; $d$ ) sensor locations; 1 vertical acceleration, 2 horizontal acceleration, 3 axial acceleration 4 Acoustic emission sensor (magnetic fastening) and, 5 Acoustic emission sensor (screw fastening) 
in Section 3 for vibration data to detect the bearing fault. The bearing experimental data is analysed with the adaptive EMD. Following the bearing fault detection model mentioned in Section 3, both normal and faulty bearing data were applied with an adaptive EMD algorithm to extract 10 IMFs for each data. In our analysis, 10 IMFs are extracted and among them, via plots of the power spectrum of each IMF, the first IMF (IMF\#1) is selected. The next step is to examine the amplitudes of the power spectrum of the first IMFs. Fig. 7 shows the plots of the power spectrum of the first IMFs of both normal and faulty bearing data. Since the power spectrum, amplitudes of high-frequency components are much smaller than those of low-frequency components, Fig. 7 is only for frequency components from $0 \mathrm{~Hz}$ to $40 \mathrm{~Hz}$. It is clearly shown that the nominal frequency (13 $\mathrm{Hz}$ ) has a very high amplitude in the bottom plot for faulty signals in comparison to the other frequency components. By contrast, the amplitude of $13-\mathrm{Hz}-$ frequency component is negligible in the plot for the normal bearing. This confirms that the proposed model using the adaptive EMD algorithm can detect the bearing fault on the outer race.
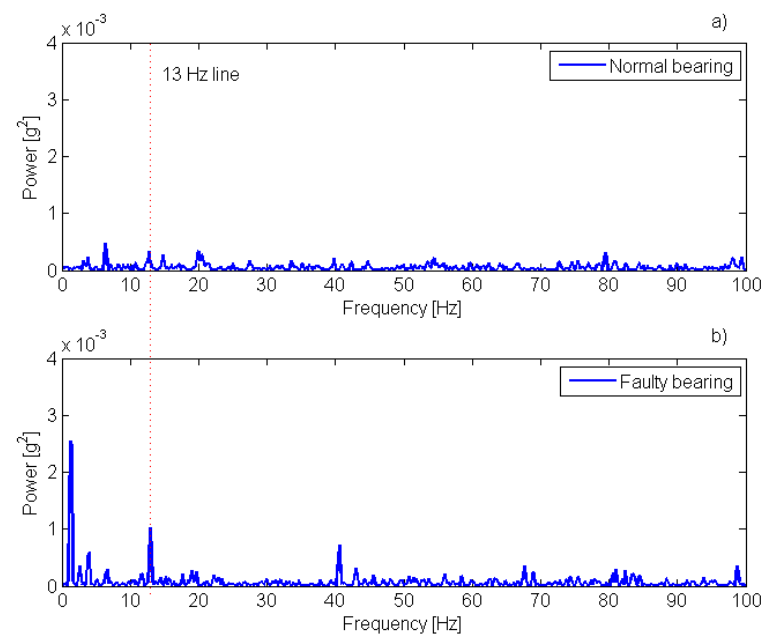

Fig. 7. Power spectrum with adaptive EMD of envelope signal of IMF \#1 of a) normal and b) faulty bearing data using proposed bearing fault detection method

Moreover, Fig. 8 shows the advantages of the proposed method over the original EMD and envelope analysis methods in terms of identifying the appearance of the faults. With the envelope analysis, the fault, which appeared in $13 \mathrm{~Hz}$, seems budding with a small peak. It is easily submerged by other peak components. The original EMD produced a better result with a higher peak, but it is not as high as the peak by the proposed method. In Fig. 8, it is clearly said that the adaptive EMD produced a better identification of the fault.
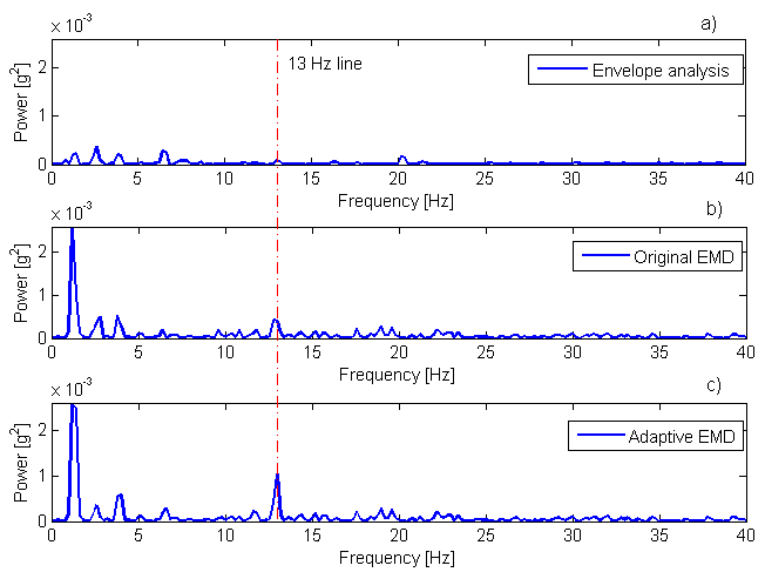

Fig. 8. Comparison of power spectrum with faulty bearing of three methods; a) envelope analysis, b) original EMD and c) AEMD

\section{CONCLUSIONS}

This paper introduces an AEMD algorithm. The advantage of the AEMD algorithm over the envelope analysis and the ensemble EMD algorithm in bearing fault detection is avoiding the fault signal energy shared among IMFs. The AEMD extracts IMFs so that the bearing fault signal will be mostly concentrated on one IMF. A performance comparison among bearing fault detection methods using envelope analysis, ensemble EMD and adaptive EMD with simulation data was carried out. It showed that the fault detection model using AEMD performs better than others. Moreover, we also validated the performance of the model with AEMD by the experimental data from practical bearing experiments. The result also indicated that the fault signature, presented by nominal frequencies, was clearly shown in the power spectrum plots.

\section{REFERENCES}

[1] Halme, J., Andersson, P. (2009). Rolling contact fatigue and wear fundamentals for rolling bearing diagnostics - state of the art. Journal of Engineering Tribology, vol. 224, no. 4, p. 377-393, DOI:10.1243/13506501JET656.

[2] Li, J. (1992). Bearing localized defect detection through wavelet decomposition of vibrations. Liang, S.Y., Wu, C.L. (eds.), Sensors and Signals Processing for Manufacturing, vol. 55, p. 187-197, American Society of Mechanical Engineers, New York.

[3] Howard, I. (1994). A Review of Rolling Element Bearing Vibration "Detection, Diagnosis and Prognosis", Aeronautical 
and Maritime Research Laboratory. Defence Science and Technology Department, Melbourne.

[4] Blau, P.J. (1992). Friction, Lubrication, and Wear Technology, ASM Handbook, vol. 18(), ASM International, Cleveland.

[5] Rai, V.K., Mohanty, A.R. (2007). Bearing fault diagnosis using FFT of intrinsic mode functions in Hilbert-Huang transform. Mechanical Systems and Signal Processing, vol. 21, no. 6, p. 2607-2615, D0l:10.1016/J.ymssp.2006.12.004.

[6] Peng, Z.K., Tse, P.W., Chu, F.L. (2005). A comparison study of improved Hilbert-Huang transform and wavelet transform: Application to fault diagnosis for rolling bearing. Mechanical Systems and Signal Processing, vol. 19, no. 5, p. 974-988, D0I:10.1016/j.ymssp.2004.01.006.

[7] Huang, N.E., Shen, Z., Long, S.R., Wu, M.C., Shih, H.H, Zheng, Q., Yen, N.C., Tung, C.C., Liu, H.H. (1998). The empirical mode decomposition and Hilbert spectrum for nonlinear and non-stationary time series analysis. Proceeding of the Royal Society A, vol. 454, no. 1971, p. 903-995, D0l:10.1098/ rspa.1998.0193.

[8] Yu, Y., Yu, D.J., Cheng, J.S. (2006). A roller bearing fault diagnosis method based on EMD energy entropy and ANN. Journal of Sound and Vibration, vol. 294, no. 1-2, p. 269-277, D0I:10.1016/j.jsv.2005.11.002.

[9] Bin, G.F., Gao, J.J., Li, X.J., Dhillon, B.S. (2012). Early fault diagnosis of rotating machinery based on wavelet packetsEmpirical mode decomposition feature extraction and neural network. Mechanical Systems and Signal Processing, vol. 27, p. 696-711, D0l:10.1016/j.ymssp.2011.08.002.

[10] Cheng, J., Yu, D., Yang, Y. (2006). A fault diagnosis approach for roller bearings based on EMD method and AR model. Mechanical Systems and Signal Processing, vol. 20, no. 2, p. 350-362, D0I:10.1016/j.ymssp.2004.11.002.

[11] Gao, Q., Duan, C., Fan, H., Meng, Q. (2008). Rotating machine fault diagnosis using empirical mode decomposition. Mechanical Systems and Signal Processing, vol. 22, no. 5, p. 1072-1081, D0l:10.1016/j.ymssp.2007.10.003.
[12] Wu, Z., Huang, N.E. (2009). Ensemble empirical mode decomposition: a noise assisted data analysis method. Advances in Adaptive Data Analysis, vol. 1, no. 1, p. 1-41, D0I:10.1142/S1793536909000047.

[13] Zhang, J., Yan, R., Gao, R.X., Feng, Z. (2010). Performance enhancement of ensemble empirical mode decomposition. Mechanical Systems and Signal Processing, vol. 24, no. 7, p. 2104-2123, DOI:10.1142/S1793536909000047.

[14] Do, V.T., Chong, U.P. (2011). Signal model-based fault detection and diagnosis for induction motors using features of vibration signal in two dimension domain. Strojniški vestnik Journal of Mechanical Engineering, vol. 57, no. 9, p. 655-666, DOI:10.5545/sv-jme.2010.162.

[15] Kahaei, H.M., Torbatian, M., Poshtan, J. (2007). Bearingfault detection using the Meyer-wavelet-packets algorithm. Strojniški vestnik - Journal of Mechanical Engineering, vol. 53, no. 3, p. 186-192.

[16] Li, Z., Ma, Z., Liu, Y., Teng, W., Jiang, R. (2015). Crack fault detection for a gearbox using discrete wavelet transform and an adaptive resonance theory neural network. Strojniški vestnik - Journal of Mechanical Engineering, vol. 61, no. 1, p. 63-73, DOI:10.5545/sv-jme.2014.1769.

[17] Lin, J., Zuo, M.J. (2003). Gearbox fault diagnosis using adaptive wavelet filter. Mechanical Systems and Signal Processing, vol. 17, no. 6, p. 1259-1269, D0l:10.1006/mssp.2002.1507.

[18] Chen, H.X., Chua, P.S.K., Lim, G.H. (2006). Adaptive wavelet transform for vibration signal modelling and application in fault diagnosis of water hydraulic motor. Mechanical Systems and Signal Processing, vol. 20, no. 8, p. 2022-2045, D0I:10.1016/J.ymssp.2005.04.007.

[19] Norton, M.P., Karczub, D.G. (2003). Fundamentals of Noise and Vibration Analysis for Engineers. Cambridge University Press, Cambridge, D0I:10.1017/СB09781139163927.

[20] Mclnerny, S.A., Dai, Y. (2003). Basic vibration signal processing for bearing fault detection. IEEE Transactions on Education, vol. 46, no. 1, p. 149-156, D0l:10.1109/TE.2002.808234. 